\title{
Influences of additives on phosphoric acid treatment of titanium dioxide as a novel white pigment
}

\author{
Aki MATSUKURA, Hiroaki ONODA* \\ Department of Informatics and Environmental Sciences, Kyoto Prefectual University, 1-5, \\ Shimogamo Nakaragi-cyo, Sakyo-ku, Kyoto 606-8522, Japan
}

Received: February 18, 2015; Revised: March 30, 2015; Accepted: April 01, 2015

(C) The Author(s) 2015. This article is published with open access at Springerlink.com

\begin{abstract}
Titanium dioxide is a photocatalytically active substance used as a white pigment for cosmetics. In sunlight, titanium dioxide catalyzes decomposition of a certain amount of sebum on the skin by ultraviolet radiation. Herein, a novel white pigment for use in cosmetics was developed from titanium dioxide and phosphoric acid at $\mathrm{pH}=4$ with and without glycerin, sodium lactate, lactic acid, and urea. The chemical composition, powder properties, photocatalytic activity, color phase, and smoothness of the samples were studied. Addition of glycerin led to a decline in the intensity of the $\mathrm{X}$-ray diffraction (XRD) peak of $\mathrm{TiO}_{2}$ relative to that of the other samples. The particle size of the materials prepared with and without additives was $0.3 \mu \mathrm{m}$; however, extensive aggregation was observed in the obtained materials. The photocatalytic activity of $\mathrm{TiO}_{2}$ was inhibited by phosphoric acid treatment. The materials prepared with sodium lactate, lactic acid, and glycerin exhibited higher photocatalytic activity than that prepared without additives. The obtained materials showed a high reflectance in the range of visible light, and the $L^{*}$ values in $L^{*} a^{*} b^{*}$ color space were high (where $L^{*}$ defines lightness, $a^{*}$ denotes the red/green value, and $b^{*}$ the yellow/blue value). These results indicate that the samples prepared in this work are suitable for use as white pigments.
\end{abstract}

Keywords: phosphoric acid; titanium dioxide; photocatalytic activity; particle size

\section{Introduction}

Titanium dioxide and zinc oxide are used as white pigments for cosmetic applications [1]. These oxides are well known to exhibit photocatalytic activity. Therefore, in their presence, a certain amount of sebum on the skin is decomposed by the ultraviolet radiation in sunlight. Several kinds of technical processes have been investigated and used to repress this effect. As one such technique, composite particles with silicon oxide have been utilized [2]. However, these materials

* Corresponding author.
E-mail: onoda@kpu.ac.jp are too harsh for use on human face. Instead, mild materials are required for use as white cosmetic pigments for facial application. In one report, it was affirmed that microfine oxide is absorbed through the skin [3]. Therefore, novel white pigments that are not absorbed by the skin must be developed.

Phosphates have been used as ceramic materials, catalysts, fluorescent materials, dielectrics, food additives, and in fuel cells, pigments, and so on $[4,5]$. Phosphate materials are well known to be non-toxic and are therefore expected to be useful as white pigments in cosmetics. White pigments with no photocatalytic activity are required for skin protection. The particle shape and size distribution of phosphate materials are important parameters for their application 
as cosmetic pigments. Spherical, homogenous particles are expected to spread well on the skin. However, overly small particles are unsuitable because the pigments might enter the pores of the skin. Generally, pigments with sub-micrometer dimensions are used for cosmetic purposes.

In earlier studies [6-8], we prepared titanium and zinc phosphate pigments with no catalytic activity; these pigments comprised spherical, homogenous particles when prepared with additives. Herein, we attempt to control the particle size by addition of glycerin, sodium lactate, lactic acid, and urea to the system of phosphoric acid and titanium dioxide.

In this study, novel white pigments are prepared from titanium dioxide and phosphoric acid at $\mathrm{pH}=4$ with and without glycerin, sodium lactate, lactic acid, and urea. The chemical composition, powder properties, photocatalytic activity, color phases, and smoothness of the obtained samples and thermal products are studied for application in cosmetics.

\section{Experimental}

Each additive (glycerin, sodium lactate, lactic acid, and urea) was added to $0.5 \mathrm{~mol} / \mathrm{L}$ in $0.5 \mathrm{~mol} / \mathrm{L}$ of phosphoric acid. The respective solutions were adjusted to $\mathrm{pH}=4$ with sodium hydroxide solution. Titanium dioxide was added to the aforementioned $0.5 \mathrm{~mol} / \mathrm{L}$ phosphoric acid solution in a molar ratio of $\mathrm{P}: \mathrm{Ti}=1: 2$. These mixtures were placed in a glass tube and shaken in hot water $\left(80{ }^{\circ} \mathrm{C}\right)$ for $1 \mathrm{~h}$. After shaking, the solutions were removed and the precipitates were washed with water and then dried at $50{ }^{\circ} \mathrm{C}$. All chemicals were of commercial purity, and were obtained from Wako Chemical Industries Ltd. (Osaka, Japan) and used without further purification.

The crystalline phase compositions of these samples were analyzed using X-ray diffraction (XRD). The XRD patterns were recorded on an X-ray diffractometer (MiniFlex, Rigaku Corp., Akishima, Japan) using monochromated $\mathrm{Cu} \mathrm{K} \alpha$ radiation. The samples were heated at $100{ }^{\circ} \mathrm{C}$ in air to remove the absorbed water. The thermal products obtained at $100{ }^{\circ} \mathrm{C}$ were characterized using scanning electron microscopy (SEM; JGM-5510LV, JEOL, Akishima, Japan). The particle size distributions of these materials were measured using a centrifugal precipitation particle-size distribution analyzer (SA-CP3L, Shimadzu Corp., Kyoto, Japan).
The cosmetic properties were evaluated in terms of the photocatalytic activity, color phase, and smoothness. The photocatalytic activity of the samples was estimated from the decomposition of methylene blue using $365 \mathrm{~nm}$ radiation [9-11]. The color of the samples was estimated based on the ultraviolet-visible (UV-Vis) reflectance spectra acquired using a UV2100, Shimadzu Corp. instrument (reference compound: $\mathrm{BaSO}_{4}$ ) and the $L^{*} a^{*} b^{*}$ values determined using a TES-135 Plus color analyzer from TES Electrical Electronic Corp. (Taipei, Taiwan, China). The particle smoothness was measured on artificial leather via KES-SE objective evaluation of the surface friction (Kato Tech Co., Ltd., Kyoto, Japan). The MIU and MMD values represent the slipping resistance (coefficient of kinetic fiction) and roughness (dispersion on coefficient of kinetic fiction) of the powders, respectively. Sample powders were spread onto the leather (sample mass: about $50 \mathrm{mg}$ ) and a sensor was then run on the powders (scan spread: $1 \mathrm{~mm} / \mathrm{s}$, area scanned: $3 \mathrm{~cm}^{2}$ ). The MIU and MMD values were respectively calculated from the power to move the sensor and the pitching of the sensor. The MIU and MMD values are dimensionless because these values are related to the coefficients of friction and scattering, respectively.

\section{Results and discussion}

\section{1 Chemical composition and powder properties}

Figure 1 shows the XRD patterns of the samples prepared with various additives followed by heating at $100{ }^{\circ} \mathrm{C}$. The anatase phase is observed in all samples. It is estimated that the phase of the samples changes from the initial $\mathrm{TiO}_{2}$ based on the changes in the intensity ratio of the peak around $25.4^{\circ}$ (Table 1 ). The decrease in the intensity of this peak is restrained in the samples prepared with sodium lactate, lactic acid, and urea. The intensity of the anatase peak in the sample prepared without additives is $80.3 \%$, and the intensity of this peak in the case of the samples prepared with sodium lactate, lactic acid, and urea is about $83 \%$. On the other hand, the intensity ratio of this peak relative to that of original $\mathrm{TiO}_{2}$ is about $71 \%$ for the sample prepared with glycerin, which is a more pronounced decrease relative to that observed for the other samples. Glycerin may easily be retained in the sample during phosphoric acid treatment because of its viscosity and 


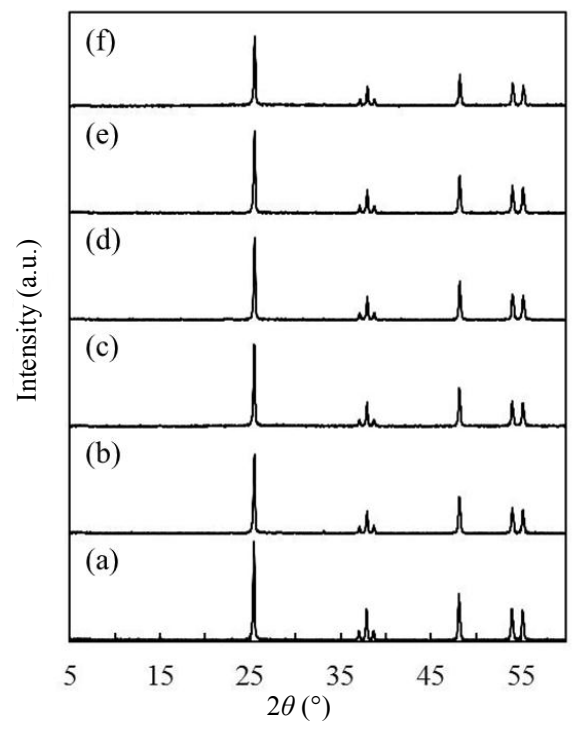

Fig. 1 XRD patterns of the samples prepared in various conditions and then heated at $100{ }^{\circ} \mathrm{C}$ : (a) $\mathrm{TiO}_{2}$, (b) no additive, (c) lactic acid, (d) urea, (e) sodium lactate, and (f) glycerin.

Table 1 XRD peak intensity, $L^{*}$ value in $L^{*} a^{*} b^{*}$ color space, and smoothness of samples prepared in various conditions and then heated at $100{ }^{\circ} \mathrm{C}$

\begin{tabular}{lccrrr}
\hline & Additive & $\begin{array}{c}\text { XRD peak (101) } \\
\text { intensity for } \mathrm{TiO}_{2}(\%)\end{array}$ & $L^{*}$ & MIU & MMD \\
\hline (a) & - & 100.0 & 99.9 & 1.28 & 0.019 \\
(b) & No & 80.3 & 99.4 & 0.79 & 0.008 \\
(c) & Lactic acid & 83.7 & 98.6 & 0.58 & 0.006 \\
(d) & Urea & 83.5 & 97.9 & 0.56 & 0.006 \\
(e) & Sodium lactate & 83.4 & 99.8 & 0.55 & 0.005 \\
(f) & Glycerin & 71.8 & 99.9 & 0.58 & 0.006 \\
\hline
\end{tabular}

high concentration. The relative intensity of the $\mathrm{TiO}_{2}$ peak decreases in the case of the sample prepared with glycerin; therefore, the XRD peaks corresponding to $\mathrm{TiO}_{2}$ become weaker than the other peaks.

Small and homogeneous particles are suitable for cosmetic applications. However, overly small particles have a major limitation in that they enter the pores of the skin [3]. Generally, pigments with sub-micrometer dimensions are used for cosmetic applications. It is difficult to specify a standard size for the white pigment particles used in cosmetics because the size of pores in the skin is affected by factors such as age, gender, and climate. Furthermore, overly large particles are inappropriate owing to the development of cracks in coatings of these particles on the skin. Thus, control of the particle size of the pigments is highly important.

Figure 2 shows the particle size distribution for the samples prepared under various conditions. Original $\mathrm{TiO}_{2}$ contains a large amount of particles with diameter

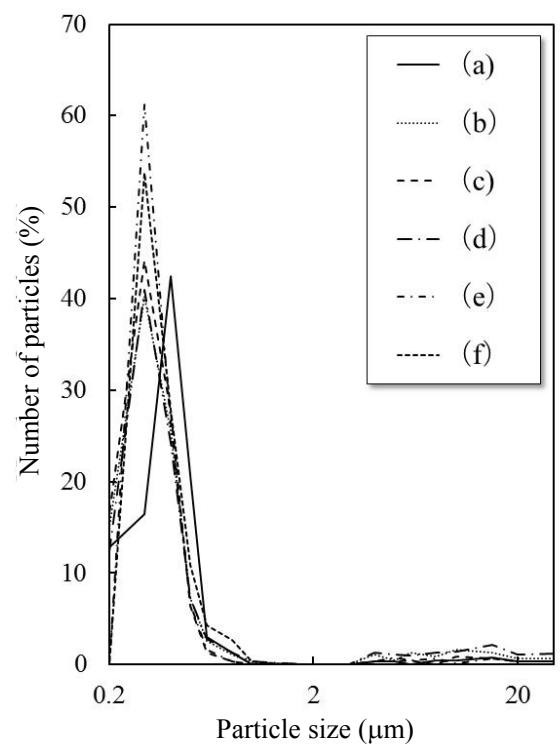

Fig. 2 Particle size distribution of samples prepared in various conditions and then heated at $100{ }^{\circ} \mathrm{C}$ : (a) $\mathrm{TiO}_{2}$, (b) no additive, (c) lactic acid, (d) urea, (e) sodium lactate, and (f) glycerin.

of $0.4 \mu \mathrm{m}$. All samples prepared herein contain a large amount of particles with diameter of $0.3 \mu \mathrm{m}$. The mode particle size for all samples prepared in this work is smaller than that of original $\mathrm{TiO}_{2}$. Furthermore, the sample prepared without additives contains $40.2 \%$ of particles with diameter of $0.3 \mu \mathrm{m}$, whereas the sample prepared with sodium lactate contains $61.2 \%$ of such particles. The particle size of the samples is thus controlled with the use of phosphoric acid treatment and additives.

In terms of particle shape, spherical particles are suitable for cosmetic applications. Figure 3 shows SEM images of the samples prepared with various additives followed by heating at $100{ }^{\circ} \mathrm{C}$. In comparison with the aforementioned particle size distribution (Fig. 2), these particles form large aggregates. Original $\mathrm{TiO}_{2}$ and all samples prepared in this work readily form large aggregates. All samples prepared herein retain this characteristic of parent $\mathrm{TiO}_{2}$. The size of the aggregates of the sample prepared without additives is about $20 \mu \mathrm{m}$, whereas the aggregates of the samples prepared with additives have dimensions of about 10 $15 \mu \mathrm{m}$ with spherical morphology. These aggregates are smaller than those of the sample prepared without additives. The aggregates of the samples prepared with sodium lactate (Fig. 3(c)) and lactic acid (Fig. 3(e)) are notably smaller than the aggregates of other samples. The sample prepared with glycerin (Fig. 3(f)) has homogenous aggregates. 

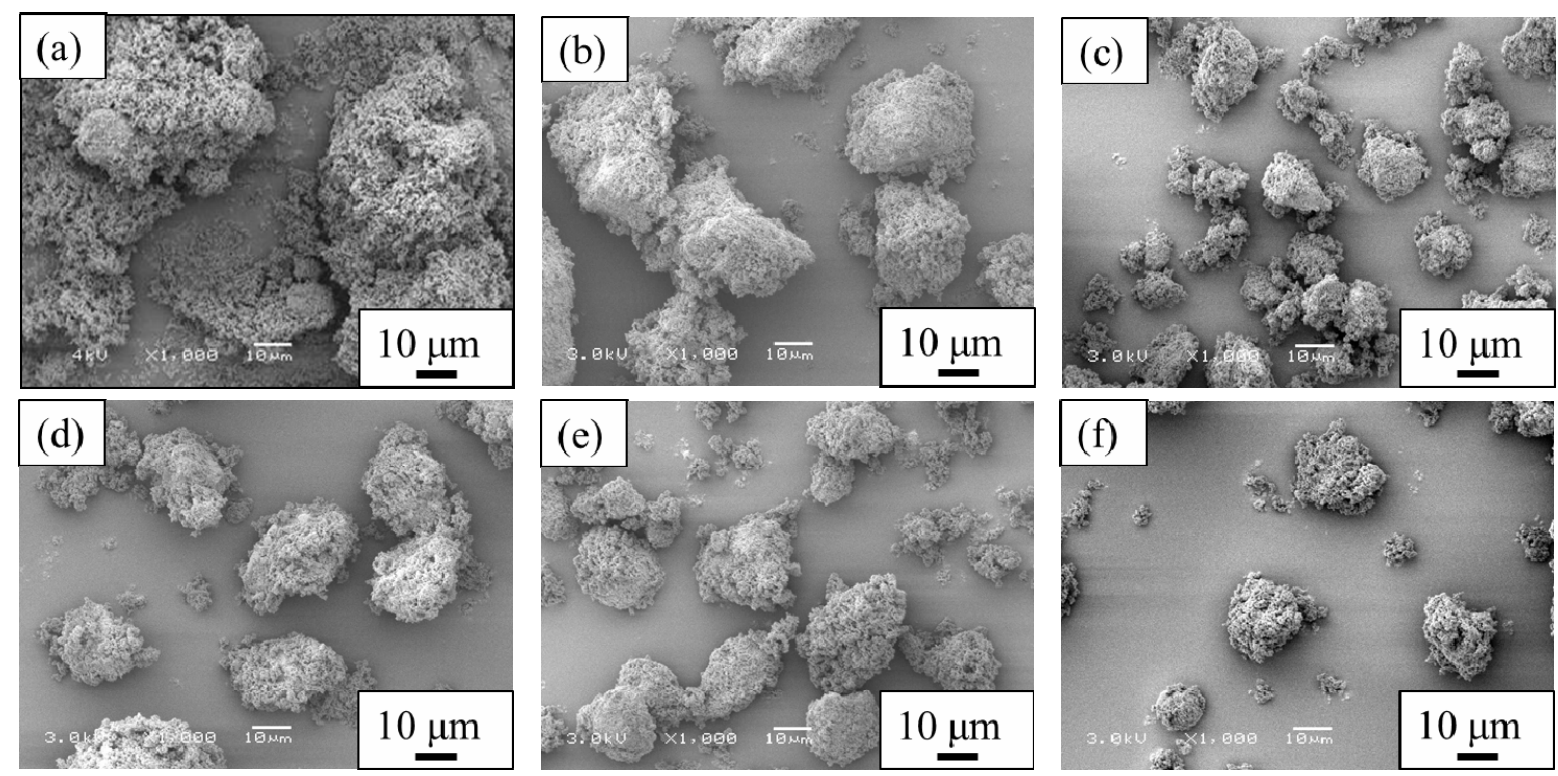

Fig. 3 SEM images of samples prepared in various conditions and then heated at $100{ }^{\circ} \mathrm{C}$ : (a) $\mathrm{TiO}_{2}$, (b) no additive, (c) lactic acid, (d) urea, (e) sodium lactate, and (f) glycerin.

\section{2 Cosmetic properties of samples}

Figure 4 presents an evaluation of the photocatalytic activity of the samples prepared under various conditions. Because $\mathrm{TiO}_{2}$ is used as a white pigment in cosmetics, this compound is evaluated for comparative analysis. Methylene blue is more extensively decomposed by $\mathrm{TiO}_{2}$ under UV irradiation than by the samples prepared in this work (Fig. 4). The photocatalytic activity of titanium dioxide is retained in the samples subjected to phosphoric acid treatment (Figs. 4(c)-4(g)). The residual ratio of absorbance is about $95 \%$ in the case of the samples prepared with urea and without additives. The samples prepared with sodium lactate, glycerin, and lactic acid exhibit higher photocatalytic activity than the sample prepared without additives. The reaction between phosphoric acid and $\mathrm{TiO}_{2}$ is influenced by the additives. Based on the XRD results presented above, the intensity of the peaks of $\mathrm{TiO}_{2}$ is lower for the sample prepared with glycerin relative to that of the samples prepared without additives and with other additives. Therefore, the photocatalytic activity of this sample is expected to be less than that of the other samples. Contrary to this expectation, the photocatalytic activity of the sample prepared with glycerin is higher than that without additives. It is difficult to clarify the relationship between the XRD peak intensity and the photocatalytic activity of the samples prepared in this work. Moreover, from the above SEM images, it is apparent that the size

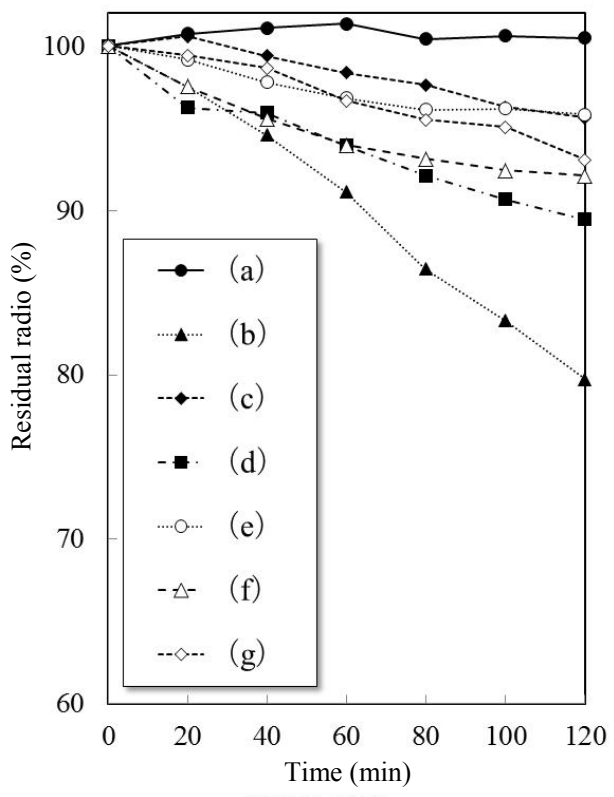

Fig. 4 Photocatalytic activity of samples prepared in various conditions and then heated at $100{ }^{\circ} \mathrm{C}$ : (a) blank, (b) $\mathrm{TiO}_{2}$, (c) no additive, (d) sodium lactate, (e) urea, (f) lactic acid, and (g) glycerin.

of the aggregates is reduced with the use of additives. However, the decrease in the photocatalytic activity does not correspond with the aggregate size. Therefore, the decreased photocatalytic activity is not caused by the reduced particle size of the aggregates.

Figure 5 shows the UV-Vis reflectance spectra of the samples prepared under various conditions and 


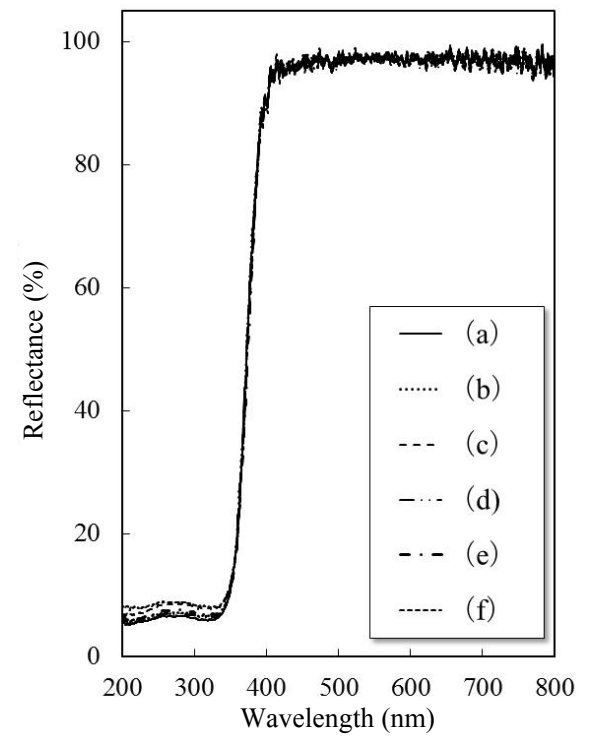

Fig. 5 UV-Vis reflectance spectra of samples prepared in various conditions and then heated at $100{ }^{\circ} \mathrm{C}$ : (a) $\mathrm{TiO}_{2}$, (b) no additive, (c) sodium lactate, (d) urea, (e) lactic acid, and (f) glycerin.

subsequently heated at $100{ }^{\circ} \mathrm{C}$. All samples have a high reflectance of more than $95 \%$ in the range of visible light in spite of phosphoric acid treatment and the use of additives. Samples prepared without heating also exhibit high reflectance (data not shown). In spite of phosphoric acid treatment and heating, the color of the samples is white. The phosphoric acid treatment and the additives have no influence on the color of the samples heated at $100{ }^{\circ} \mathrm{C}$ and those not subjected to heating.

Table 1 shows the $L^{*} a^{*} b^{*}$ values of $\mathrm{TiO}_{2}$ and the samples prepared under various conditions followed by thermal treatment at $100{ }^{\circ} \mathrm{C}$. The values of $a^{*}$ and $b^{*}$ are similar for all samples (not shown); therefore, we focus on the value of $L^{*}$. The $L^{*}$ value of all samples is over 97 and is sufficiently high in spite of phosphoric acid treatment with additives. Thus, all samples are white powders that are suitable for use as white pigments for cosmetic applications. These results are in accord with the previously discussed UV-Vis reflectance data.

As mentioned above, a pigment with high smoothness spreads well on the skin; therefore, powder smoothness is also an important parameter for use in cosmetics [12]. Table 1 shows the smoothness of the samples prepared under various conditions. Generally, for cosmetic applications, the suitable MIU and MMD values are smaller than 0.6 and below 0.04 , respectively. The MIU values of the samples prepared via phosphoric acid treatment and additives decrease relative to those of original $\mathrm{TiO}_{2}$. The MIU value of the sample prepared without additives is 0.79 , whereas the MIU values of the samples prepared with various additives are in the range of 0.57 . The MMD values of the samples prepared under the various conditions are smaller than that of $\mathrm{TiO}_{2}$. It is found that the various additives produce samples with high smoothness. Based on the data in Fig. 3, the samples prepared with additives have smaller aggregates than $\mathrm{TiO}_{2}$ and the sample prepared without additives. The MIU and MMD values may be influenced by this aggregation.

\section{Conclusions}

Novel white pigments were obtained from phosphoric acid and titanium dioxide with the addition of sodium lactate, urea, lactic acid, and glycerin. Phosphoric acid treatment with additives resulted in a decline of the intensity of the XRD peaks relative to that of $\mathrm{TiO}_{2}$. The particle size of the obtained materials prepared with and without additives was $0.3 \mu \mathrm{m}$, which is smaller than the particle size of original $\mathrm{TiO}_{2}$. The particle size was successfully controlled by phosphoric acid treatment. Furthermore, large aggregates were formed in the obtained materials due to retention of the tendency towards aggregation of the parent $\mathrm{TiO}_{2}$. The use of additives induced a decrease in the size of the aggregates relative to the size of the aggregates formed by parent $\mathrm{TiO}_{2}$. The photocatalytic activity of $\mathrm{TiO}_{2}$ was inhibited by phosphoric acid treatment. The materials prepared with sodium lactate, lactic acid, and glycerin had higher photocatalytic activity than that prepared without additives. It was proposed that the reaction between phosphoric acid and $\mathrm{TiO}_{2}$ is disrupted by these additives. The materials prepared without heating and with heating at $100{ }^{\circ} \mathrm{C}$ had high reflectance in the range of visible light, and the $L^{*}$ values in $L^{*} a^{*} b^{*}$ color space of the obtained materials were high. These results confirmed that the samples prepared in this work were white powders. Finally, the samples prepared with additives are more promising as white pigments for cosmetics than that prepared without additives.

\section{Acknowledgements}

The authors are grateful to Dr. Takeshi Toyama, Nihon University, Japan, for assistance with smoothness 
measurements.

Open Access: This article is distributed under the terms of the Creative Commons Attribution License which permits any use, distribution, and reproduction in any medium, provided the original author(s) and the source are credited.

\section{References}

[1] Diebold U. The surface science of titanium dioxide. Surf Sci Rep 2003, 48: 53-229.

[2] Senzui M, Tamura T, Miura K, et al. Study on penetration of titanium dioxide $\left(\mathrm{TiO}_{2}\right)$ nanoparticles into intact and damaged skin in vitro. J Toxicol Sci 2010, 35: 107-113.

[3] Gamer AO, Leibold E, van Ravenzwaay B. The in vitro absorption of microfine zinc oxide and titanium dioxide through porcine skin. Toxicol in Vitro 2006, 20: 301-307.

[4] Jones DJ, Aptel G, Brandhorst M, et al. High surface area mesoporous titanium phosphate: Synthesis and surface acidity determination. $J$ Mater Chem 2000, 10: 1957-1963.

[5] Bhaumik A, Inagaki S. Mesoporous titanium phosphate molecular sieves with ion-exchange capacity. $J$ Am Chem Soc 2001, 123: 691-696.

[6] Onoda H, Yamaguchi T. Influence of ultrasonic treatment on preparation and powder properties of titanium phosphate. J Mater Chem 2012, 22: 19826-19830.

[7] Onoda H, Yamaguchi T, Takenaka A. Synthesis and pigmental properties of titanium phosphate with the addition of urea. Int J Cosmetic Sci 2012, 34: 86-90.

[8] Onoda H, Haruki M, Toyama T. Preparation and powder properties of zinc phosphates with additives. Ceram Int 2014, 40: 3433-3438.

[9] Ramaswamy V, Jagtap NB, Vijayanand S, et al. Photocatalytic decomposition of methylene blue on nanocrystalline titania prepared by different methods. Mater Res Bull 2008, 43: 1145-1152.

[10] Du P, Bueno-López A, Verbaas M, et al. The effect of surface $\mathrm{OH}$-population on the photocatalytic activity of rare earth-doped $\mathrm{P} 25-\mathrm{TiO}_{2}$ in methylene blue degradation. $J$ Catal 2008, 260: 75-80.

[11] Mills A. An overview of the methylene blus ISO test for assessing the activities of photocatalytic films. Appl Catal B: Environ 2012, 128: 144-149.

[12] Cheng SY, Yuen CWM, Kan CW, et al. A comprehensive study of silicone-based cosmetic textile agent. Fiber Polym 2009, 10: 132-140. 